OPEN ACCESS

Edited by:

Qihui Zhou,

Qingdao University, China

Reviewed by:

Xinghong Zhao,

Sichuan Agricultural University, China

Chunxiong Zheng,

Sun Yat-sen University, China

*Correspondence:

Yizhou Zhan

zhanyz@ucas.ac.cn

Tieli Zhou

wyztli@163.com

Jianming Cao

wzcjming@163.com

Specialty section:

This article was submitted to

Nanoscience,

a section of the journal

Frontiers in Chemistry

Received: 14 October 2021 Accepted: 03 November 2021 Published: 25 November 2021

Citation:

Lin $Y$, Zhang $Y$, Liu S, Ye D, Chen L, Huang $N$, Zeng $W$, Liao W, Zhan $Y$, Zhou T and Cao J (2021) Quercetin Rejuvenates Sensitization of Colistin-

Resistant Escherichia coli and Klebsiella Pneumoniae Clinical Isolates

to Colistin.

Front. Chem. 9:795150.

doi: 10.3389/fchem.2021.795150

\section{Quercetin Rejuvenates Sensitization of Colistin-Resistant Escherichia coli and Klebsiella Pneumoniae Clinical Isolates to Colistin}

\author{
Yishuai Lin ${ }^{1}$, Ying Zhang ${ }^{1}$, Shixing Liu ${ }^{2}$, Dandan Ye $^{2}$, Liqiong Chen ${ }^{1}, \mathrm{Na}$ Huang $^{2}$, \\ Weiliang Zeng ${ }^{1}$, Wenli Liao ${ }^{2}$, Yizhou Zhan ${ }^{3 *}$, Tieli Zhou ${ }^{2 *}$ and Jianming Cao ${ }^{1 *}$ \\ ${ }^{1}$ School of Laboratory Medicine and Life Science, Wenzhou Medical University, Wenzhou, China, ${ }^{2}$ Department of Clinical \\ Laboratory, the First Affiliated Hospital of Wenzhou Medical University, Wenzhou, China, ${ }^{3}$ Engineering Research Center of Clinical \\ Functional Materials and Diagnosis \& Treatment Devices of Zhejiang Province, Wenzhou Institute, University of Chinese Academy \\ of Sciences; Oujiang Laboratory (Zhejiang Lab for Regenerative Medicine, Vision and Brain Health), Wenzhou, China
}

Colistin is being considered as "the last ditch" treatment in many infections caused by Gram-negative stains. However, colistin is becoming increasingly invalid in treating patients who are infected with colistin-resistant Escherichia coli (E. coli) and Klebsiella Pneumoniae (K. pneumoniae). To cope with the continuous emergence of colistin resistance, the development of new drugs and therapies is highly imminent. Herein, in this work, we surprisingly found that the combination of quercetin with colistin could efficiently and synergistically eradicate the colistin-resistant $E$. coli and $K$. pneumoniae, as confirmed by the synergy checkboard and time-kill assay. Mechanismly, the treatment of quercetin combined with colistin could significantly downregulate the expression of $m c r-1$ and $m g r B$ that are responsible for colistin-resistance, synergistically enhancing the bacterial cell membrane damage efficacy of colistin. The colistin/quercetin combination was notably efficient in eradicating the colistin-resistant $E$. coli and $K$. pneumoniae both in vitro and in vivo. Therefore, our results may provide an efficient alternative pathway against colistinresistant $E$. coli and $K$. pneumoniae infections.

Keywords: Escherichia coli, klebsiella pneumoniae, quercetin, synergy mechanism, colistinresistance

\section{INTRODUCTION}

Antibiotics undertake a significant duty in terms of decreasing the morbidity and mortality associated with bacteria-induced infections. Nevertheless, the overuse and abuse of antibiotics have brought out the emergence of resistant bacteria (Arias and Murray, 2012). Besides, the development of novel antibiotics is considerably slow and challenging, which increased the therapeutic difficulty for infections caused by drug-resistant bacterial infections (Cordoba et al., 2015). To address this issue, new antibacterial materials and the combination of non-antibacterial and antibiotics have been regarded as a new treatment strategy in overcoming the drug resistance of bacteria (Li et al., 2020; Su et al., 2020).

As a cationic antimicrobial peptide, colistin exploits the antibacterial effect through interplaying with the lipid A section of lipopolysaccharide (LPS) and destroys the Gram-negative bacteria outer membrane afterward (Phan et al., 2017; Shen et al., 2018; Tran et al., 2018). Despite colistin remaining high-efficient in antimicrobial activity, the massive use of colistin as a clinical therapeutic 
TABLE 1 | The MIC values against the 12 clinical isolates.

\begin{tabular}{|c|c|c|c|c|c|c|c|c|c|c|c|}
\hline \multirow[t]{2}{*}{ Species } & \multirow[t]{2}{*}{ Strains $^{a}$} & \multicolumn{10}{|c|}{ MIC values ${ }^{b}(\mu \mathrm{g} / \mathrm{ml})$} \\
\hline & & ATM & CAZ & FEP & IPM & CIP & LVX & GEN & TOB & COL & Que \\
\hline \multirow[t]{6}{*}{ E. coli } & DC90 & $\geq 32^{R}$ & $\geq 32^{R}$ & $\geq 32^{R}$ & $\geq 8^{R}$ & $\geq 2^{R}$ & $\geq 4^{\mathrm{R}}$ & $\geq 16^{\mathrm{R}}$ & $\geq 16^{R}$ & $\geq 4^{R}$ & $\geq 32$ \\
\hline & DC3411 & $\geq 32^{R}$ & $\geq 32^{R}$ & $\geq 32^{R}$ & $\leq 2$ & $\geq 2^{R}$ & $\geq 4^{\mathrm{R}}$ & $\leq 4$ & $\leq 4$ & $\geq 4^{R}$ & $\geq 32$ \\
\hline & DC3539 & $\geq 32^{R}$ & $\geq 32^{R}$ & $\geq 32^{R}$ & $\leq 2$ & $\geq 2^{R}$ & $\geq 4^{\mathrm{R}}$ & $\geq 16^{R}$ & $\geq 16^{R}$ & $\geq 4^{R}$ & $\geq 32$ \\
\hline & DC3737 & $\geq 32^{R}$ & $\geq 32^{R}$ & $\geq 32^{R}$ & $\leq 2$ & $\geq 2^{R}$ & $\geq 4^{\mathrm{R}}$ & $\geq 16^{\mathrm{R}}$ & $\leq 4$ & $\geq 4^{\mathrm{R}}$ & $\geq 32$ \\
\hline & DC3806 & $\geq 32^{R}$ & $\geq 32^{R}$ & $\geq 32^{R}$ & $\leq 2$ & $\geq 2^{R}$ & $\geq 4^{\mathrm{R}}$ & $\geq 16^{R}$ & $\geq 16^{R}$ & $\geq 4^{R}$ & $\geq 32$ \\
\hline & DC3846 & $\geq 32^{R}$ & $\geq 32^{R}$ & $\geq 32^{R}$ & $\leq 2$ & $\geq 2^{R}$ & $\geq 4^{\mathrm{R}}$ & $\geq 16^{\mathrm{R}}$ & $\geq 16^{\mathrm{R}}$ & $\geq 4^{\mathrm{R}}$ & $\geq 32$ \\
\hline \multirow[t]{6}{*}{ K. pneumoniae } & FK610 & $\geq 32^{R}$ & $\geq 32^{R}$ & $\geq 32^{R}$ & $\geq 8^{R}$ & $\geq 2^{R}$ & $\geq 4^{\mathrm{R}}$ & $\geq 16^{\mathrm{R}}$ & $\geq 16^{R}$ & $\geq 4^{\mathrm{R}}$ & $\geq 32$ \\
\hline & FK1913 & $\geq 32^{R}$ & $\geq 32^{R}$ & $\geq 32^{R}$ & $\leq 2$ & $\leq 0.5$ & $\leq 1$ & $\leq 4$ & $\leq 4$ & $\geq 4^{R}$ & $\geq 32$ \\
\hline & FK2066 & $\leq 8$ & $\leq 8$ & $\leq 8$ & $\leq 2$ & $\leq 0.5$ & $\leq 1$ & $\leq 4$ & $\leq 4$ & $\geq 4^{R}$ & $\geq 32$ \\
\hline & FK4134 & $\leq 8$ & $\geq 32^{R}$ & $\geq 32^{R}$ & $\leq 2$ & $\geq 2^{R}$ & $\geq 4^{\mathrm{R}}$ & $\geq 16^{\mathrm{R}}$ & $\geq 16^{R}$ & $\geq 4^{R}$ & $\geq 32$ \\
\hline & FK6663 & $\geq 32^{R}$ & $\geq 32^{R}$ & $\geq 32^{R}$ & $\geq 8^{R}$ & $\geq 2^{R}$ & $\geq 4^{R}$ & $\geq 16^{R}$ & $\geq 16^{R}$ & $\geq 4^{\mathrm{R}}$ & $\geq 32$ \\
\hline & FK6696 & $\geq 32^{R}$ & $\geq 32^{R}$ & $\geq 32^{R}$ & $\geq 8^{R}$ & $\geq 2^{R}$ & $\geq 4^{\mathrm{R}}$ & $\geq 16^{\mathrm{R}}$ & $\geq 16^{R}$ & $\geq 4^{R}$ & $\geq 32$ \\
\hline
\end{tabular}

${ }^{a}$ Strain numbers in bold indicate they are multi-drug resistant.

${ }^{b}$ Values in bold indicate bacterial resistance.

GNB, Gram-negative bacteria; ATM, aztreonam; CAZ, ceftazidime; FEP, cefepime; IMP, imipenem; CIP, ciprofloxacin; LVX, levofloxacin; GEN, gentamicin; TOB, tobramycin; COL, colistin; Que, Quercetin.

and forage additive has led to the emergence of colistin resistance (Liu et al., 2016; Xavier et al., 2016). Since colistin has been regarded as 'the last ditch' treatment for the infections caused by Gram-negative pathogens, it is highly desired to develop novel strategies to overcome the colistin resistance in bacteria or to resensitize the bacteria to colistin treatment.

Quercetin is a flavanol compound which is widely existed in the plant kingdom and has a variety of biological activities such as antibacterial and anti-inflammatory efficiency (Vilaca et al., 2012). Previous studies have shown that the combination of quercetin with either tetracycline or meropenem can destroy the cellular integrity and downregulate the expression of the drug-resistant genes in E. coli (Pal and Tripathi, 2019; Qu et al., 2019). Meanwhile, similar results were found in the combination of quercetin with other antimicrobials such as amikacin, tobramycin, levofloxacin, ceftriaxone, and gentamycin (Pal and Tripathi, 2020; Vipin et al., 2020). However, whether quercetin is efficient in overcoming the colistin resistance in bacteria, especially clinical isolates, remains undiscovered.

Herein, the synergism of the colistin/quercetin combination in eradicating the 12 colistin-resistant E. coli and K. pneumoniae clinical isolates was studied. The antimicrobial efficiency of the colistin/quercetin combination was studied both in vitro and in vivo. Also, the expression of the genes related to drug resistance is determined in bacteria after the colistin/quercetin treatment to elucidate the mechanism of quercetin in alleviating the colistin resistance in the tested clinical isolates.

\section{MATERIALS AND METHODS}

\section{Bacterial Strains and Chemicals}

The twelve non-duplicated colistin-resistant $E$. coli and $K$. pneumoniae clinical isolates (see Table 1) were recovered from the First Affiliated Hospital of Wenzhou Medical University from 2016 to 2018 . All isolates were identified by matrix-assisted laser desorption/ionization time-of-flight mass spectrometry (MALDI-TOF/MS; bioMérieux, Lyons, France). Tested strains were frozen in Luria Bertani (LB) broth medium (Sigma-Aldrich, Saint Louis, United States) containing $30 \%$ glycerol at $-80^{\circ} \mathrm{C}$ before use.

Quercetin was purchased from MedChem Express (MCE) Co., Ltd. (New Jersey, United States), and all antibiotics used in this study, including colistin, aztreonam, ceftazidime, cefepime, imipenem, ciprofloxacin, levofloxacin, gentamicin, tobramycin, and amikacin were purchased from Wenzhou Kangtai Biological Technology Co., Ltd. (Zhejiang, China).

\section{Determination of Antimicrobial Resistance Profiles}

Minimum inhibitory concentrations (MICs) of commonly used antibiotics, colistin, and quercetin were judged by the broth microdilution in cation-adjusted Mueller-Hinton Broth (CAMHB) (Thermo Fisher Scientific, America). The breakpoint scoring was interpreted according to the Clinical and Laboratory Standards Institute (CLSI, 2020) for both E. coli and K. pneumoniae. Briefly, in the 96-well plates, bacterial suspension $\left(5 \times 10^{5} \mathrm{CFU} / \mathrm{ml}\right)$ was mixed with CAMHB medium containing drugs with concentrations ranging from $0.016-32 \mu \mathrm{g} / \mathrm{ml}$. The plates were incubated at $37^{\circ} \mathrm{C}$ overnight. The MIC was defined as the lowest drug concentration that completely inhibited the development of the microorganism, observed by the naked eye. The breakpoint of antibiotics was interpreted accordingly to the CLSI. Each MIC test against all isolates was performed in duplicate and repeated three times.

\section{Synergism Testing}

The checkerboard assay was conducted to explore the synergy effect between colistin and quercetin via determining the fractional inhibitory concentration (FIC) index (Yu et al., 2020). Briefly, colistin was continuously dilute 2 -fold along the 
TABLE 2 | Summary of MIC values and $\mathrm{FICl}$ of colistin combined with quercetin against the 12 colistin-resistant $E$. coli and $K$. pneumoniae clinical isolates.

\begin{tabular}{|c|c|c|c|c|c|c|}
\hline \multirow[t]{2}{*}{ Strain } & \multicolumn{2}{|c|}{$\begin{array}{c}\text { Antimicrobial } \\
\text { susceptibility (MIC, } \\
\mu \mathrm{g} / \mathrm{mL})\end{array}$} & \multicolumn{4}{|c|}{ Antimicrobial combination (MIC, $\mu \mathrm{g} / \mathrm{mL}$ ) } \\
\hline & CST & Que & CST + Que & $\mathbf{F I C l}$ & Potentiation & Interpretation \\
\hline DC90 & 64 & 256 & $2+32$ & 0.4388 & 32-fold & synergy \\
\hline DC3411 & 4 & 256 & $1+128$ & 0.75 & 4-fold & additive \\
\hline DC3539 & 4 & 256 & $1+32$ & 0.375 & 4-fold & synergy \\
\hline DC3737 & 8 & 512 & $2+64$ & 0.375 & 4-fold & synergy \\
\hline DC3806 & 4 & 256 & $1+32$ & 0.75 & 4-fold & additive \\
\hline DC3846 & 8 & 256 & $2+128$ & 0.375 & 4-fold & synergy \\
\hline FK610 & 16 & 256 & $4+64$ & 0.50 & 4-fold & synergy \\
\hline FK1913 & 64 & 256 & $8+16$ & 0.3125 & 8 -fold & synergy \\
\hline FK2066 & 32 & 512 & $16+128$ & 0.75 & 2-fold & additive \\
\hline FK4134 & 8 & 256 & $2+32$ & 0.375 & 4-fold & synergy \\
\hline FK6663 & 32 & 256 & $8+32$ & 0.375 & 4-fold & synergy \\
\hline FK6696 & 32 & 256 & $16+64$ & 0.75 & 2-fold & additive \\
\hline
\end{tabular}

$x$-axis, while quercetin was continuously diluted 2 -fold along the $y$-axis to establish a matrix in 96-well plates, where each well contained different concentrations of two drugs. The bacterial suspension $(100 \mu \mathrm{L})$ was added to each well to reach an ultimate bacterial concentration of approximately $5 \times 10^{5}$ colony-forming unit $(\mathrm{CFU}) / \mathrm{mL}$. Bacteria in the absence of antibiotic exposure served as the control. MIC was taken as the lowest concentration of antibiotics that can inhibit the growth of bacteria.

The combination inhibition effect for each strain was confirmed by through the FIC index: $\mathrm{FIC}_{\text {colistin }}=\mathrm{MIC}_{\text {colistin }}$ in combination $/ \mathrm{MIC}_{\text {colistin }}$ alone; $\mathrm{FIC}_{\text {quercetin }}=\mathrm{MIC}_{\text {quercetin }}$ in combination/ $\mathrm{MIC}_{\text {quercetin }}$ alone; FIC index $=\mathrm{FIC}_{\text {colistin }}+$ FIC $_{\text {quercetin. }}$ Synergy judgment criteria were: FICI $\leq 0.5$ indicated synergism; $0.5<\mathrm{FICI} \leq 1$ indicated additive effect; $1<$ FICI $\leq 2$ indicated irrelevant effect; FICI $>2$ indicated antagonistic effect (Qu et al., 2019).

\section{Time-Kill Assay}

The time-kill assay was carried out according to a published experimental process (Berard et al., 2016) with minor modifications. Briefly, colistin-resistant E. coli $(n=6)$ or $K$. pneumoniae $(n=6)\left(1 \times 10^{6} \mathrm{CFU} / \mathrm{ml}\right)$ were inoculated in $10 \mathrm{ml}$ CAMHB containing colistin ( $1 / 2 \mathrm{MIC})$, quercetin $(1 / 2$ MIC), or quercetin $(1 / 2 \mathrm{MIC})+$ colistin $(1 / 2 \mathrm{MIC})$. Bacteria in the CAMHB medium without antibiotics treatment served as the control. The tubes were incubated at $37^{\circ} \mathrm{C}$. At various time points $(0,2,4,6,12$, and $24 \mathrm{~h})$ post-incubation, an aliquot $(100 \mu \mathrm{L})$ of bacterial suspension was withdrawn and spotted on MuellerHinton agar plates, incubated overnight at $37^{\circ} \mathrm{C}$, followed by $\mathrm{CFU}$ counting (Zhang et al., 2019). A decrease $\geq 3 \log 10$ for CFU/mL by $24 \mathrm{~h}$ was defined as the bactericidal activity, and a decrease $\geq 2$ $\log 10$ for $\mathrm{CFU} / \mathrm{mL}$ was defined as synergistic activity calculated by comparison of two drugs combination and single drug treatment (Berard et al., 2016).

\section{Quantitative Real-Time PCR (qRT-PCR)}

Quantification of expression of $m c r-1$ and $m g r B$ genes in E. coli and $K$. pneumoniae respectively were performed using reverse transcription PCR (qRT-PCR) (Jayol et al., 2014; Gaudereto et al., 2019). 16SrRNA and $r p o B$ served as standardized reference genes for E. coli and K. pneumoniae, respectively (Tadesse et al., 2012; Liu et al., 2017). Primers were composited by Shanghai Huada Biological Engineering Co., Ltd (Shenzhen, China). A commercial RNA extraction kit was used to isolate total RNA from the strains cultured in LB broth containing quercetin ( $1 / 2 \mathrm{MIC})$, colistin $(1 / 2$ $\mathrm{MIC})$, or quercetin $(1 / 2 \mathrm{MIC})+$ colistin $(1 / 2 \mathrm{MIC})$ for $16-18 \mathrm{~h}$ (Tiangen Biotech, Beijing, China) according to the user's guideline (Bulman et al., 2017). Quantified gene expression by the $\Delta \Delta \mathrm{Ct}$ method. Experiments for each gene were performed in triplicate.

\section{Bacterial Cell Permeability}

To study the damage of various antibiotics treatments on the bacterial cell membrane, E. coli and K. pneumoniae $\left(\mathrm{OD}_{600}=0.5\right)$ were inoculated into LB broth containing quercetin ( $1 / 2 \mathrm{MIC})$, colistin $(1 / 2 \mathrm{MIC})$, and quercetin $(1 / 2 \mathrm{MIC})+$ colistin $(1 / 2 \mathrm{MIC})(\mathrm{Qu}$ et al., 2019), followed by incubation in a shaker $(180 \mathrm{rpm})$ at $37^{\circ} \mathrm{C}$ for $24 \mathrm{~h}$. Then, these suspensions were centrifuged at 5,000 rpm for $5 \mathrm{~min}$. The supernatant was collected and subjected to the alkaline phosphatase (ALP) activity test via a corresponding kit (Solarbio, Beijing). All experiments were performed in triplicate. In general, AKP/ALP uses $p$-nitrophenyl phosphate (pNPP) as a phosphatase substrate which turns yellow $\left(\lambda_{\max }=405 \mathrm{~nm}\right)$ when dephosphorylated by ALP.

Similarly, the $\beta$-galactosidase activity in the supernatant was tested via its corresponding kit (Solarbio, Beijing). $\beta$-galactosidase decomposes $\quad p$-nitrobenzene- $\beta$-D-galactopyranoside into $p$-nitrophenol, and the activity of $\beta$-galactosidase is calculated by measuring its absorbance at $420 \mathrm{~nm}$.

\section{Scanning Electron Microscope (SEM)}

To study the morphological changes of the bacterial cell membrane after various treatments, SEM images of bacteria were taken (Watanabe et al., 2018). Briefly, a glass slide $(24 \times$ $24 \mathrm{~mm}$ in size) was plated in each well of a six-well plate, then, bacteria $\left(1.5 \times 10^{8} \mathrm{CFU} / \mathrm{ml}\right)$ in $2 \mathrm{ml} \mathrm{MH}$ broth containing 


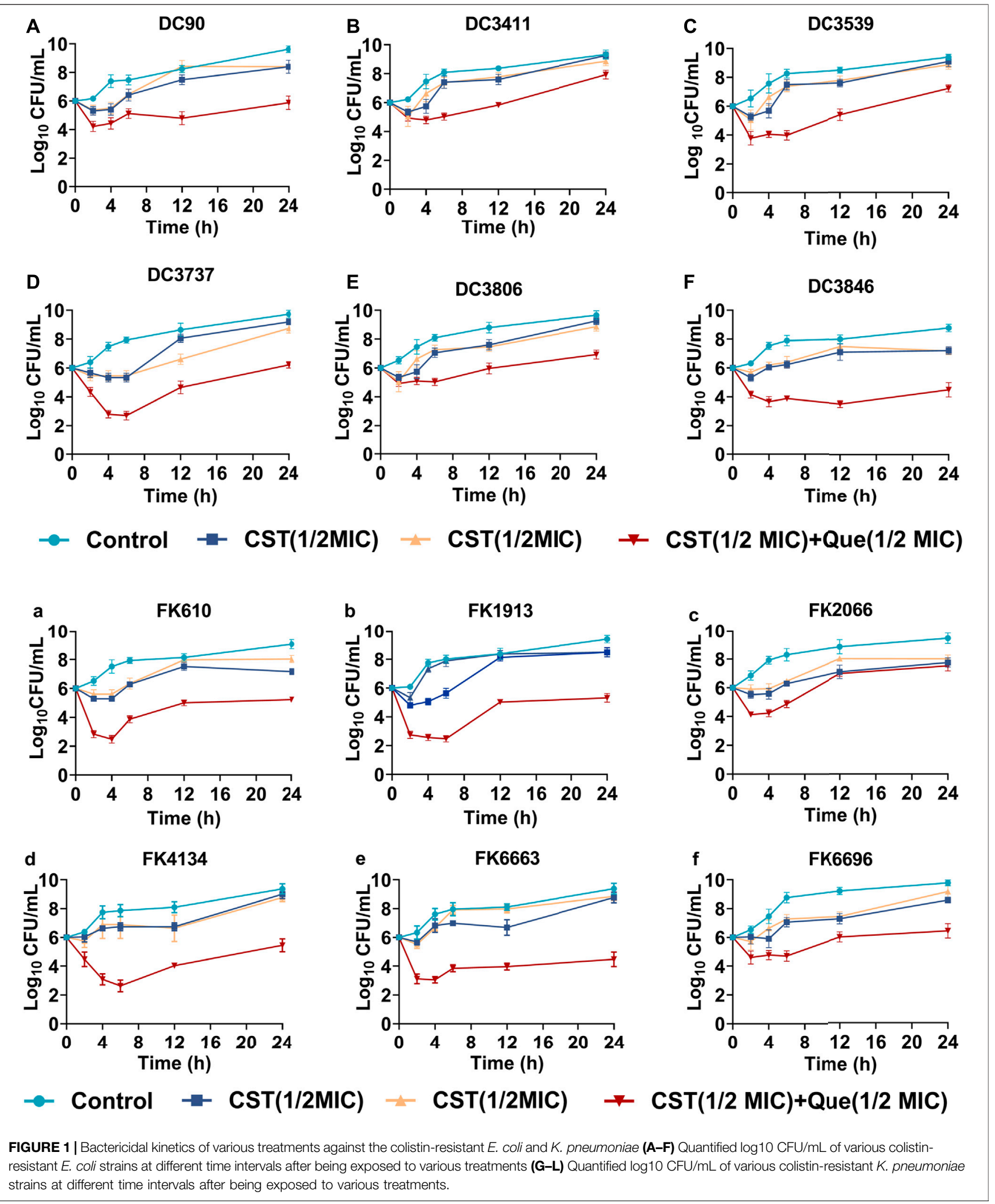



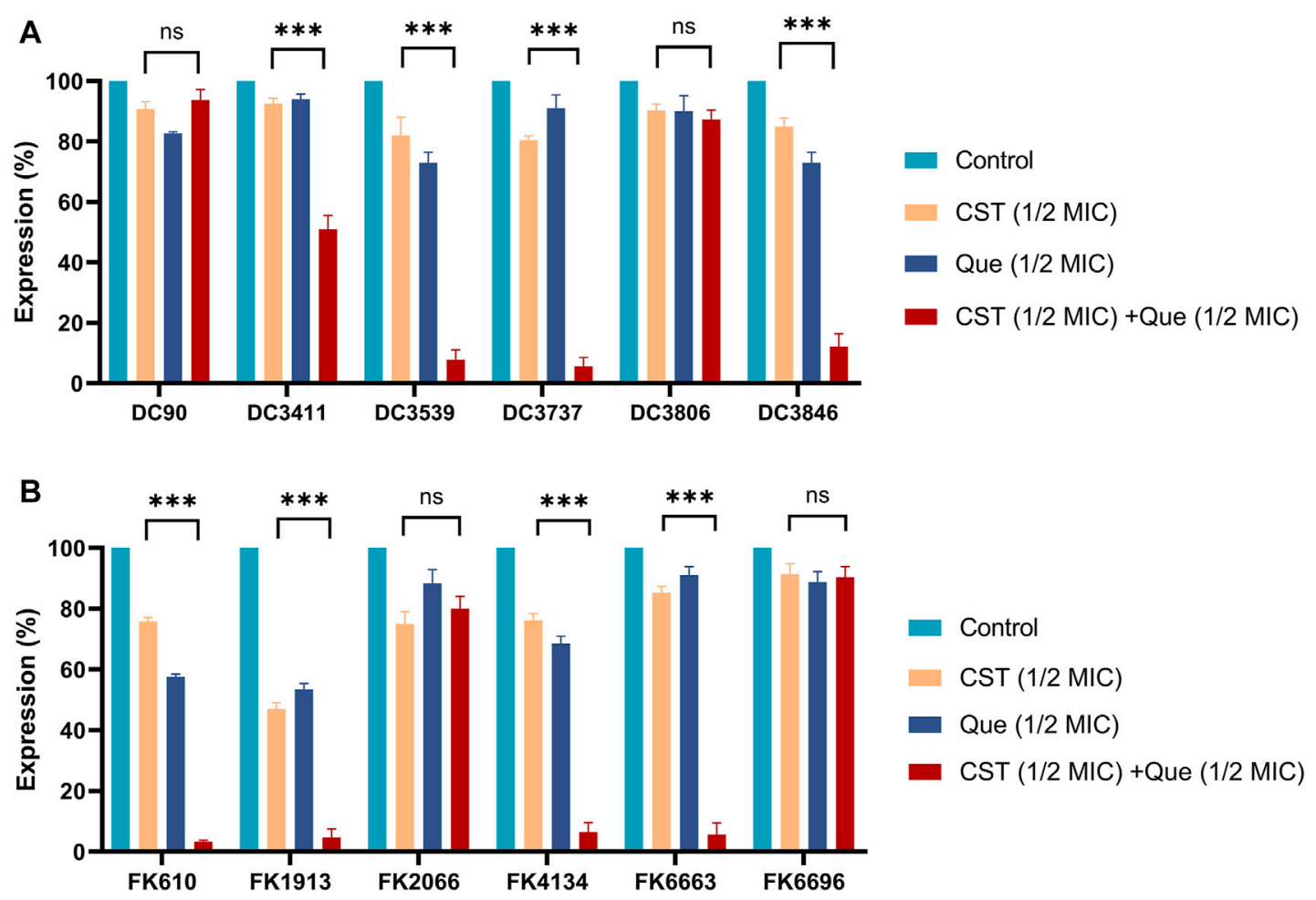

FIGURE 2 Combination of colistin with quercetin significantly downregulates the mRNA levels of $m c r-1$ and $m g r B$ genes in $E$. coli and $K$. pneumoniae, respectively (A) Relative mRNA levels of $m c r-1$ in different $E$. coli strains after the various treatments (B) Relative mRNA levels of mgrB in different $K$. pneumoniae strains after the various treatments. 16srRNA and rpoB were used as the internal reference for $E$. coli and $K$. pneumoniae, respectively, and the mRNA levels of those two genes were taken as $100 \%$. All experiments were performed in triplicate. The results were displayed as means \pm standard deviations over the three independent experiments. ns stands for not significant, ${ }^{\star} p<0.05,{ }^{\star \star} p<0.01,{ }^{\star \star \star} p<0.001$, determined by the Student's $t$-test.

quercetin ( $1 / 2$ MIC), colistin ( $1 / 2$ MIC), or quercetin $(1 / 2$ MIC)+colistin ( $1 / 2 \mathrm{MIC})$ were added to each well. The plate was incubated at $37^{\circ} \mathrm{C}$ for $18 \mathrm{~h}$. Subsequently, the glass slides were taken out, washed three times with PBS. The remaining bacteria were fixed in $500 \mu \mathrm{L} 5 \%(\mathrm{v} / \mathrm{v})$ glutaraldehyde (Solarbio, Beijing) and incubated overnight at $4^{\circ} \mathrm{C}$. After washing three times in $1 \times \mathrm{PBS}$, the cells were treated with air drying and platinum coating, then observed by SEM (S-3000N, Japan).

\section{Eradication of Bacterial Infection in vivo}

To establish the murine infection model, specific-pathogen-free (SPF) female ICR (Institute of Cancer Research) mice, 5-6 weeks old (Charles River, Hangzhou, China), were used. Mice were raised according to the National Standards for Laboratory Animals of China (GB 14925-2010). All animal experiments were approved by the Zhejiang Association for Science and Technology SYXK (ID: SYXK (Zhejiang)2018-0017) and carried out according to the guideline of the Wenzhou Laboratory Animal Welfare and Ethics.

In short, mice were intraperitoneally injected with cyclophosphamide (Solarbio, Beijing) at 4 days $(150 \mathrm{mg} / \mathrm{kg}$ ) and one day $(100 \mathrm{mg} / \mathrm{kg})$ before thigh intramuscular injection to induce a neutropenia model $\left(\leq 100\right.$ neutrophils $\left./ \mathrm{mm}^{3}\right)$. Then mice were divided into four groups ( 3 mice per group), each posterior thigh muscle of mice was exponentially injected with $100 \mu \mathrm{L}$ of bacterial suspension $\left(1.5 \times 10^{7} \mathrm{CFU} / \mathrm{ml}\right)$. At $2 \mathrm{~h}$ post bacterial inoculation, mice were administered with 1) $1 \times \mathrm{PBS}$ (untreated group), 2) quercetin (50 mg/kg), 3) colistin/quercitin combination $(5 \mathrm{mg} / \mathrm{kg}$ colistin $+50 \mathrm{mg} / \mathrm{kg}$ quercetin, or $7.5 \mathrm{mg} / \mathrm{kg}$ colistin $+50 \mathrm{mg} / \mathrm{kg}$ quercetin) by intraperitoneal injection. At $24 \mathrm{~h}$ post-treatment, mice were euthanized through cervical dislocation. The posterior thigh tissue was collected, homogenized, diluted in PBS, spread on agar plates, and cultured at $37^{\circ} \mathrm{C}$ overnight for CFU quantification.

\section{Statistical Analysis}

With regard to all analyses, a $p$ value $<0.05$ was defined to be statistically significant. Paired two-tailed $t$-test and one-way ANOVA were performed to compared changes in gene expression level, cell membrane, and in vivo bacterial colony counts. All statistically calculated values were carried out with SPSS v.22.0 software (SPSS Inc, Chicago, IL, United States).

\section{Ethics}

The patients provided their written informed consent to participate in this study. The study and consent procedure was 

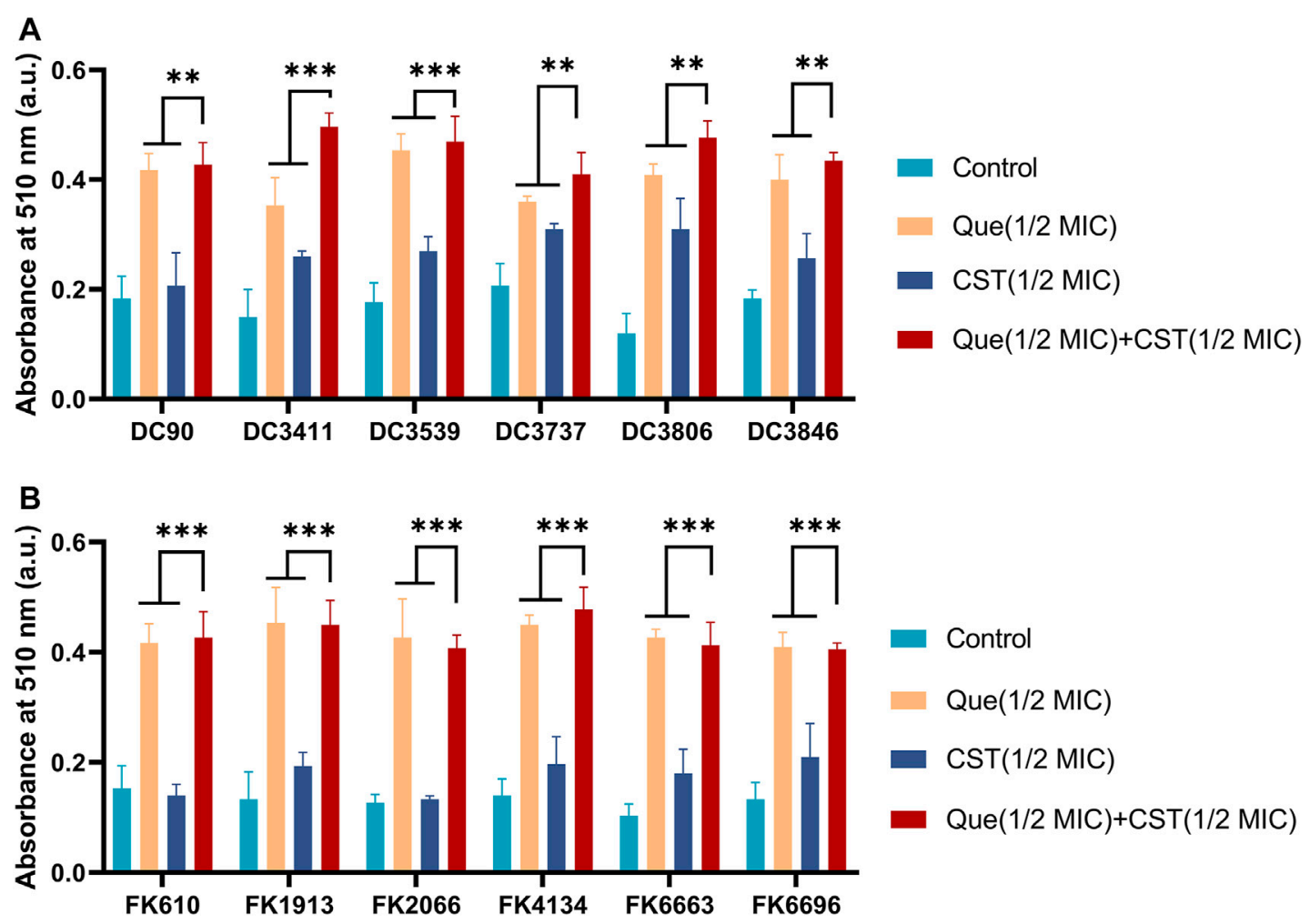

FIGURE 3 | Combination of quercetin and colistin contributes to the damage of bacterial cell membranes and the leakage of bacterial ALPs. The absorbance at $510 \mathrm{~nm}$ integrity of $E$. coli (A) and K. pneumoniae (B) by detecting ALP. The chart indicates that an increase for alkaline phosphatase in the medium after exposure to the specified compound. The results are shown as the mean and standard deviation of three independent experiments. ns stands for not significant, ${ }^{\star} p<0.05$, ${ }^{\star \star} p<0.01$, ${ }^{\star \star *} p<0.001$, determined by the Student's $t$-test.

approved by the ethics committee of the First Affiliated Hospital of Wenzhou Medical University (No. 2020140).

\section{RESULTS}

\section{Susceptible Testing of Antimicrobial Resistance Profiles}

The MIC of several commonly used antibiotics and quercetin were tested against the clinically isolated $E$. coli and $K$. pneumoniae, as well as E. coli ATCC 25922 (drug-susceptible control). As shown in Table 1, the MIC value of colistin was tested to be $4-32 \mu \mathrm{g} / \mathrm{ml}$ for the majority of the tested $E$. coli and $K$. pneumoniae strains. Of note, $1 E$. coli strain, DC90, and $1 K$. pneumoniae strain, FK 1913, were found extremely resistant to colistin treatment (MIC $\geq 64 \mu \mathrm{g} / \mathrm{ml}$ ). These results suggest the colistin resistance of those clinically isolated $E$. coli and $K$. pneumoniae strains. Moreover, these clinical isolates were resistant to the majority of the commercially available antibiotics as exemplified in Table 1. Ten strains showed a multidrug-resistant phenotype. Specifically, all the six E. coli strains were tested resistant to the treatment of aztreonam or cefepime and $5 \mathrm{~K}$. pneumoniae strains were resistant to cefepime. Imipenem still holds therapeutic efficacy to 5 E. coli strains and three $K$. pneumoniae strains. The MIC values of the quality control strains are all within the acceptable range.

\section{Synergism Test by the Checkerboard Method}

The checkerboard method was applied to test the synergism between colistin and quercetin against the 12 clinical isolates. As shown in Table 2, a 4-32-fold decrease in MIC of colistin for four E. coli (DC90, DC3539, DC3737, and DC3846) and four $K$. pneumoniae (FK610, FK 1913, FK4134, and FK6663) was observed when colistin was used in combination with quercetin. For those strains, the FIC values were calculated to be 0141-0.375, suggesting an excellent synergism between colistin and quercetin. The quercetin/colistin combination exhibited the additive effect $(\mathrm{FIC}=0.75)$ against two $E$. coli (DC3411, DC3806) and two K. pneumoniae (FK 2066, FK6696) strains.

\section{Time-Kill Assay}

Time-kill assay was carried out to further demonstrate the synergistic drug combination of quercetin and colistin against the clinical isolates. As shown in Figure 1, in the absence of antibiotic or quercetin exposure (control group), bacteria grew 

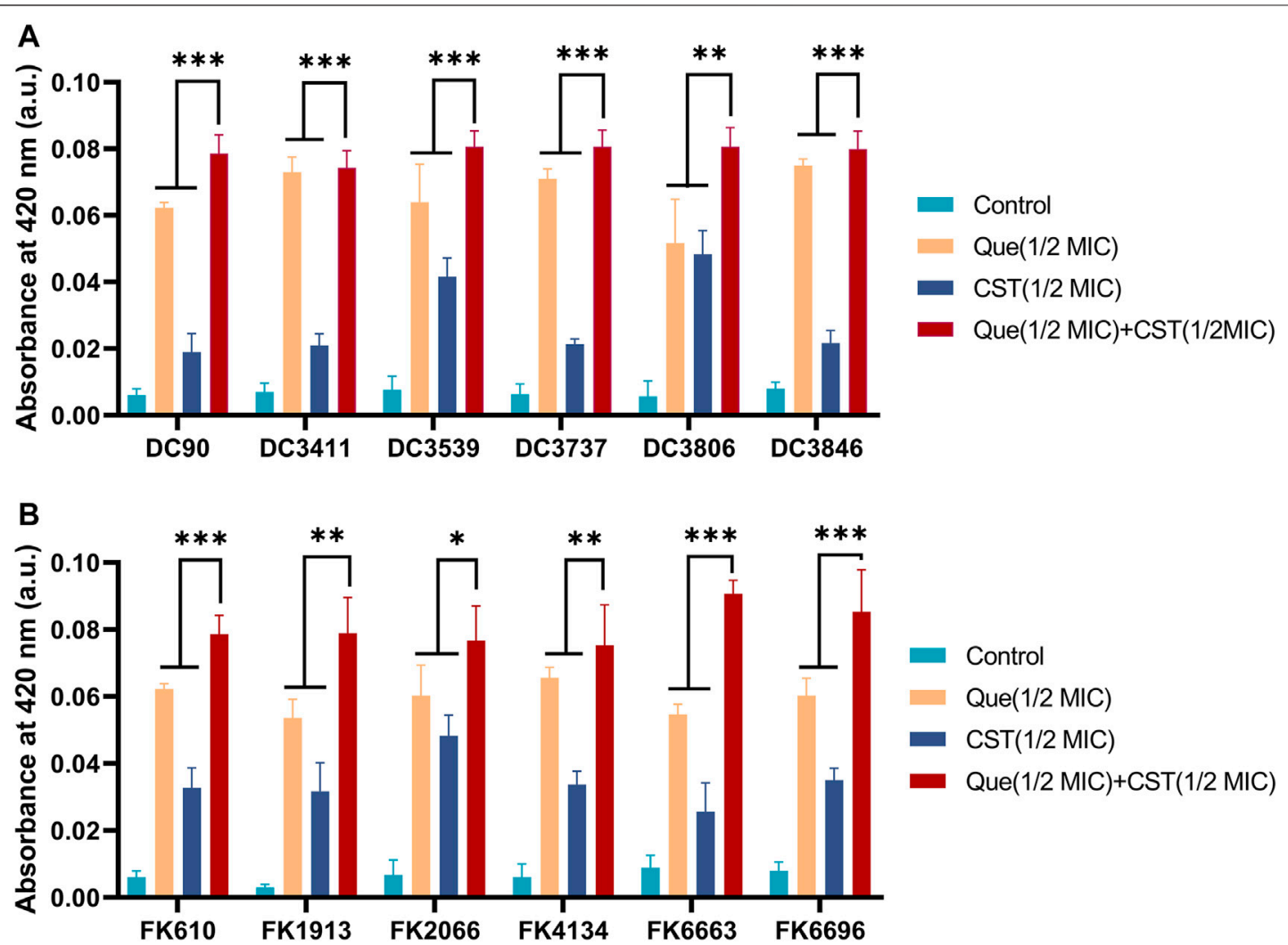

FIGURE 4 | Combination of quercetin and colistin contributes to the damage of bacterial cell membranes and the leakage of bacterial $\beta$-galactosidases. Quercetin and colistin combine to disrupt bacterial cell wall integrity of $E$. coli (A) and $K$. pneumoniae (B) by detecting the release of intracellular $\beta$-galactosidase. The chart indicates that an increase for $\beta$-galactosidase in the medium after exposure to the specified compound. The results are shown as the mean and standard deviation of three independent experiments. ns stands for not significant, ${ }^{\star} p<0.05,{ }^{\star \star} p<0.01,{ }^{\star \star \star} p<0.001$, determined by the Student's $t$-test.

rampantly in a conventional gradient growth trend. The treatment of either colistin or quercetin at a concentration of $1 / 2$ of its corresponding MIC slightly inhibited the bacterial growth within the first $6 \mathrm{~h}$, after which the bacterial growth was negligibly affected, and there is no notable difference between the drug-treated groups and the control group after $24-\mathrm{h}$ incubation. Of note, the colistin/quercetin combination was efficient in eradicating those clinical isolates. The majority of the bacteria were killed (a reduction of $2-4$ in $\log 10 \mathrm{CFU} / \mathrm{mL}$ ) by the colistin/ quercetin combination within the first $6 \mathrm{~h}$, after which the bacterial growth was still largely inhibited. As a result, a reduction of $2-4$ in $\log 10 \mathrm{CFU} / \mathrm{mL}$ was observed in the bacterial suspension treated with the colistin/quercetin combination compared with the bacteria in the control group, regardless of the bacterial strains.

\section{Gene Expression}

To figure out the detailed mechanism of the colistin/quercetin combination, the gene expression of $m c r-1$ in E. coli and $m g r B$ in $K$. pneumoniae after various treatments was studied using qRTPCR. As illustrated in Figure 2, the treatment of either colistin or quercetin alone could inhibit up to $12 \%$ of $m c r-1$ expression in E. coli and up to $50 \%$ of $m g r B$ expression in K. pneumoniae. The colistin/quercetin combination exhibited notably higher efficiency in suppressing the expression of $m c r-1$ and $m g r B$, a up to $90 \%$ reduction of $m c r-1$ expression in E. coli, and up to $95 \%$ reduction of $m g r B$ expression in K. pneumoniae was observed in the majority of the clinical isolates. Of special note, in strains such as E. coli DC3806 and K. pneumoniae FK6696, the colistin/ quercetin combination did not induce the significant gene suppression efficiency compared to the monotherapy of either colistin or quercetin.

\section{Permeability of Bacterial Cell Membrane}

Subsequently, the permeability of the bacterial cell membrane after various treatments was investigated. Extracellular ALPs and $\beta$-galactosidases after various treatments were determined using their corresponding assays. As shown in Figures 3, 4, upon the treatment of quercetin, the amount of extracellular ALPs and $\beta$-galactosidases was notably enhanced in both $E$. coli and $K$. pneumoniae, suggesting the damage of the bacterial cell membrane. Although colistin also triggered a notable ALPs release from bacterial cells, its efficiency was not comparable to that of quercetin. Furthermore, the colistin/quercetin combination induced similar or better bacterial cell membrane damage efficiency compared to quercetin treatment alone in all the tested isolates. 

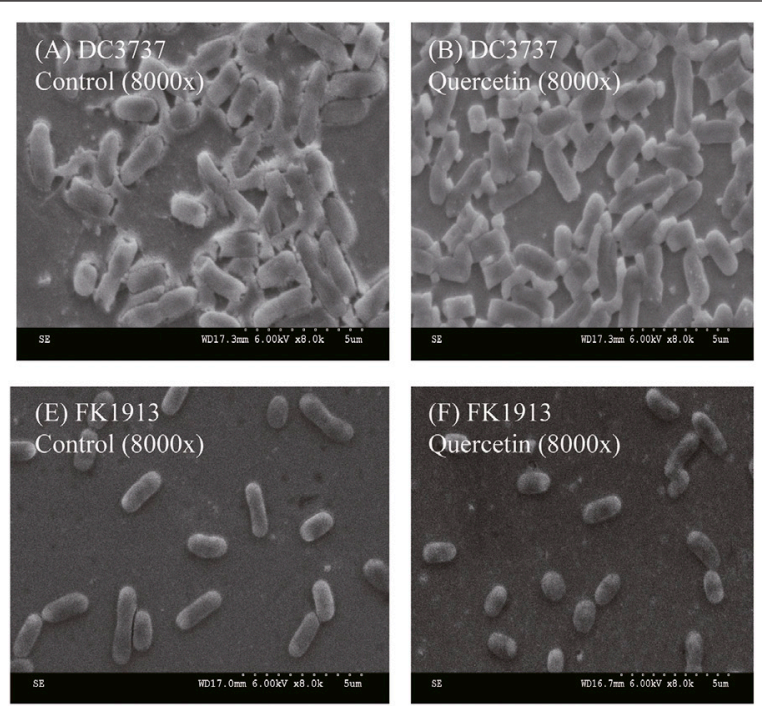
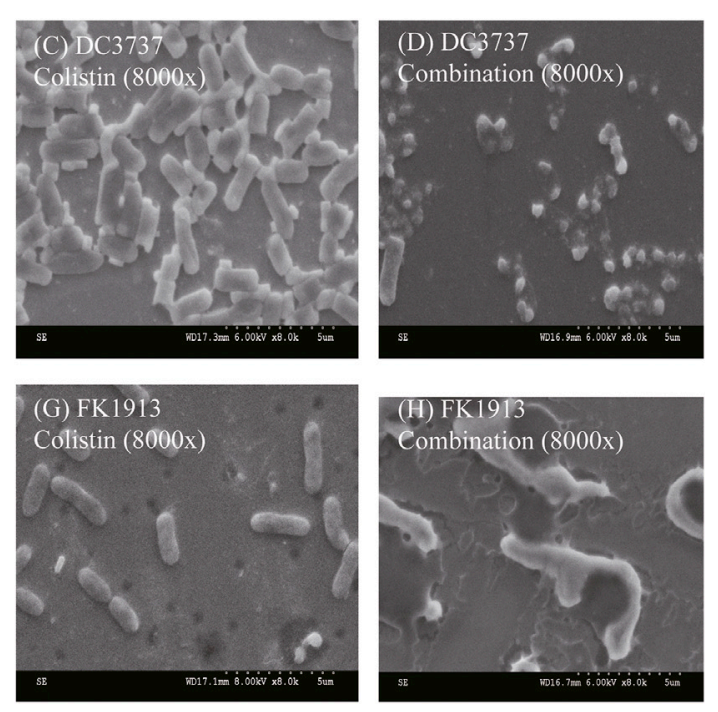

FIGURE 5 I SEM images showing the morphological change of bacterial cell membrane of E. coli DC3737 and K. pneumoniae FK1913 after various treatments.

\section{Scanning Electron Microscope (SEM)}

Furthermore, the change of bacterial morphologies after various treatments was investigated via SEM. The colistin/quercetin combination induced significant bacterial cell membrane damage. Whereas the cell morphology of bacteria after the treatment of quercetin or colistin at its corresponding FIC concentration was similar to that of the PBS-treated bacteria.

\section{In vivo Bactericidal Efficacy Elevation}

Since we have demonstrated that colistin combined with quercetin has a synergistic bactericidal effect in vitro, it is still important to verify the effectiveness of the combination through in vivo experiments, especially animal models. Herein, a murine model with $E$. coli and $K$. pneumoniae infection was established and received various treatments. As shown in Figure 6, monotherapy treatment with quercetin at a dose of $50 \mathrm{mg} / \mathrm{kg}$ only slightly inhibited the growth of E. coli DC1913 and K. pneumoniae FK3737, as illustrated by a reduction of 1.01 and 0.16 in $\log _{10}$ CFU/thigh in E. coli DC1913 and K. pneumoniae FK3737, respectively, compared with the PBS-treated mice. The treatment of colistin at a dose of $5 \mathrm{mg} / \mathrm{kg}$ every $24 \mathrm{~h}$ only slightly inhibited the bacterial growth with a reduction of 0.08-0.51 in $\log _{10}$ CFU/thigh in E. coli DC 1913- or K. pneumoniae FK3737infected mice. However, a higher dose of $7.5 \mathrm{mg} / \mathrm{kg}$ could efficiently enhance the therapeutic performance of colistin, yielding an $\sim 1.06$ reduction in $\log _{10} \mathrm{CFU} /$ thigh in the infected mice. Particularly, the combination of quercetin $(50 \mathrm{mg} / \mathrm{kg})$ and colistin $(5 \mathrm{mg} / \mathrm{kg})$ achieved a $\log 10$ reduction of 1.522 for E. coli DC1913 and 1.438 for K. pneumoniae FK3737, respectively, compared to the PBS-treated mice. Moreover, dose-dependent bacterial eradication efficacy was observed, as illustrated by a $\log 10$ reduction of 2.304 for E. coli DC1913 and 2.017 for K. pneumoniae FK3737 was achieved when the infected mice were treated with quercetin $(50 \mathrm{mg} / \mathrm{kg})$ and colistin $(7.5 \mathrm{mg} / \mathrm{kg})$. These results collectively suggest the colistin/quercetin combination is efficient in eradicating the colistin-resistant $E$. coli and $K$. pneumoniae infections in vivo, posing a great potential in clinical translation.

\section{DISCUSSION}

Colistin has been considered as the "last-line" therapy against infections caused by multidrug-resistant Gram-negative pathogens (Shen et al., 2020). Previous studies and clinical practice have shown that colistin may cause nephrotoxicity and neurotoxicity (Gregoire et al., 2017). Therefore, WHO has listed colistin as one of the critically vital antibiotics that need to be replaced (Biswas et al., 2014). As a result, the clinical use of colistin was discontinued and replaced with other effective antibiotics. However, the increasing challenges caused by multi-drug resistant Gram-negative bacteria, together with the lack of new type antibiotics against such strains have made colistin come back into the spotlight (Liu et al., 2021; Su et al., 2021). Worse still, many clinically isolated pathogens are resistant to colistin treatment. Therefore, there is a dire need to develop new techniques to overcome the drug resistance of bacteria against colistin or to make the resistant bacteria resensitize to colistin treatment again. In this research, we developed a novel strategy to rejuvenate the power of colistin against the colistinresistant clinical isolates E. coli and K. pneumoniae via the combination of colistin and quercetin.

Quercetin is a flavonol compound that widely exists in edible plants with low toxicity to humans and animals and can be used for therapeutic purposes as an eatables supplement ( 3 times a day, $250-500 \mathrm{mg}$ each time). Of note, quercetin can scavenge free radicals to prevent lipid peroxidation, inhibiting tumor proliferation (Reyes-Farias and Carrasco-Pozo, 2019). Also, it has been reported that the combination of quercetin with other antibiotics such as vancomycin and tetracycline possess 


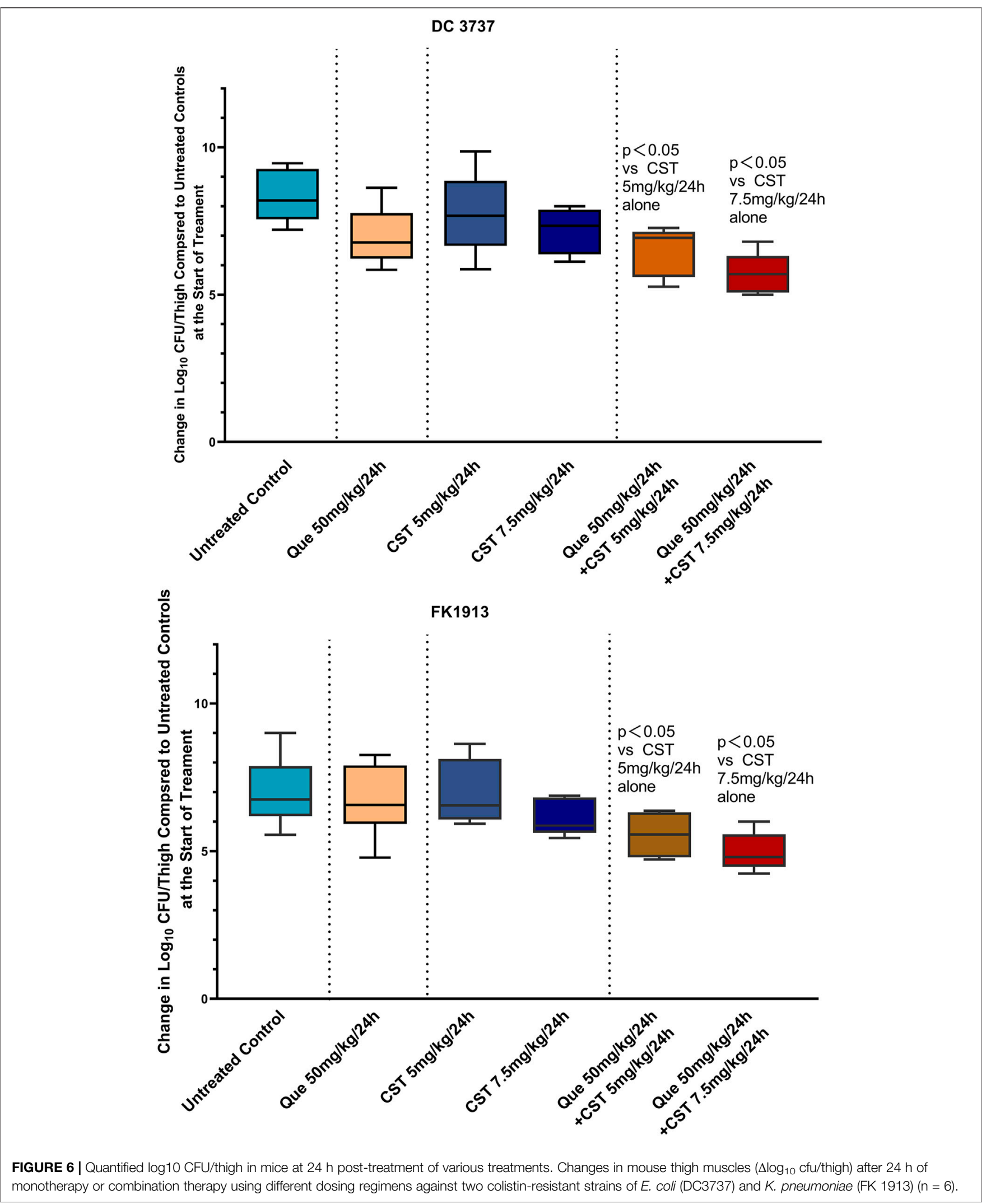




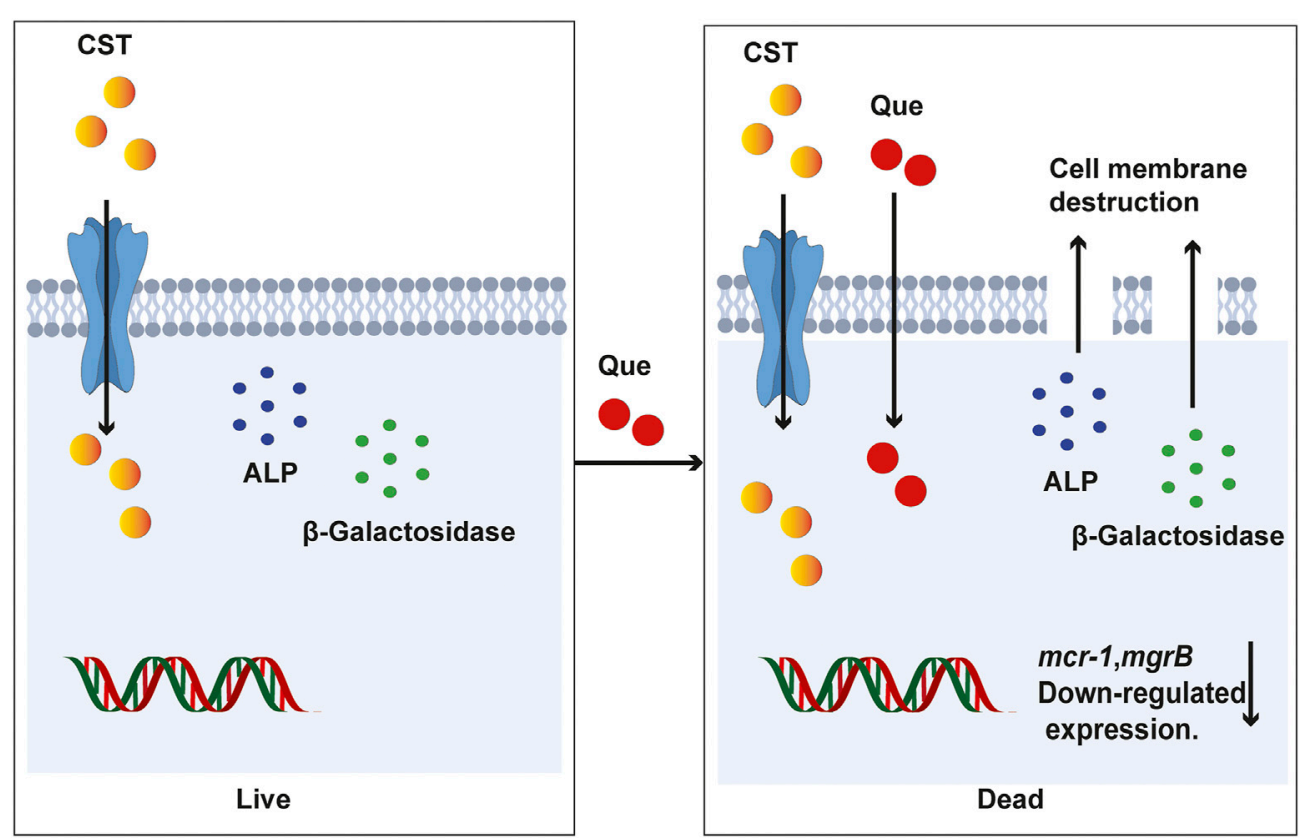

FIGURE 7 | A proposed mechanism of quercetin/colistin combination in eradicating colistin-resistant E. coli and K. pneumoniae.

synergistic antibacterial activity against E. coli and Staphylococcus aureus (Qu et al., 2019; Yu et al., 2020). In addition, quercetin possesses low cytotoxicity and can increase intracellular oxidative stress in tumor cells usually at concentrations $>40 \mathrm{mM}$ (Vargas and Burd, 2010). However, the effect of quercetin on the antimicrobial efficacy of colistin has never been shown.

In this work, we presented the first report of the synergistic activity between quercetin and colistin against the colistinresistant E. coli and K. pneumoniae clinical isolates. The MIC of colistin was reduced by $2-32$-fold when it combined with quercetin, suggesting that quercetin and colistin combination therapy could be a promising treatment option to confront the infections caused by colistin-resistant E. coli and K. pneumoniae. We also carried out the time-killing assays to evaluate in vitro antibacterial activity of the quercetin/colistin combination. We confirmed that the quercetin/colistin combination is efficient to eradicate the colistin-resistant E. coli and K. pneumoniae. Besides, the previous study has found out that the expression of $m c r-1$ and $m g r B$ genes in colistin-resistant E. coli and K. pneumoniae are upregulated (Liao et al., 2020). $m c r-1$ is a plasmid-mediated E. coli resistance gene, which is widely spread in the world to cause colistin resistance (Jiang et al., 2020). When it comes to $K$. pneumoniae, $m g r B$ gene is considered to be a common cause of colistin resistance. After using the combination of quercetin and colistin, we noticed that the mRNA levels of $m c r-1$ and $m g r B$ genes were significantly decreased in most strains (Figure 2). Of note, the genes of interest were not down-regulated in several strains, probably due to the differences between strains and the complexity of colistin resistance mechanism, which could be a promising topic for our future studies.
Similar to many other natural extracts, quercetin can exert antibacterial activity to a certain extent (Jaisinghani, 2017; Hao et al., 2021; Zhu et al., 2021). Although these natural extracts may differ in their antimicrobial mechanism, most of them induce bacterial lysis via cell membrane damage. For example, baicalein, another flavonoid, can disturb cell wall integrity by binding peptidoglycan directly (Zhao et al., 2001). This phenomenon was also observed in our experiment where intercellular bacterial ALPs and $\beta$-galactosidases were released after the treatment of quercetin/colistin combination (Figures 3, 4). Furthermore, we observed the morphological change of bacterial envelopes after the treatment of quercetin/colistin combination using a scanning electron microscope (Figure 5). Taken together, these results indicated that the combination therapy could efficiently destroy the integrity of the bacterial cell membrane.

Encouraged by the excellent synergism of quercetin/colistin combination, we further constructed the murine infection model and evaluated the antimicrobial efficacy of quercetin/colistin combination in vivo. Notably, colistin combined with quercetin showed that the efficacy is higher than monotherapy treatment, with 1.8-3.2 $\log 10 \mathrm{CFU}$ reduction after $24 \mathrm{~h}$ (Figures 6A,B), showing the significant antibacterial effect in vivo against $m c r-1$ producing $E$. coli and $m g r B$ producing $K$. pneumoniae colistin-resistant isolates. This provides a practical foundation for clinical therapy. Considering that experimental conditions cannot be completely simulated in vivo, such as dynamic antibiotic variety, bacterial virulence, and host immune response against infection (Zhou et al., 2017), more efforts should be made in the future to elucidate the abovementioned mechanisms or issues. 
In summary, the combination of quercetin and colistin provides a new treatment choice for the infections caused by colistin-resistant bacteria by reducing the dosage and concentration of colistin. Quercetin could significantly resensitize the colistin-resistant E. coli and K. pneumoniae clinical isolates to colistin in vitro. In addition, results indicate that quercetin combined with colistin act synergistically to damage the cell membrane and wall permeability and induce an effective reduction in the expression of $\mathrm{mcr}-1$ and $m g r B$ genes (Figure 7). In the murine infection model caused by the colistin-resistant E. coli and $K$. pneumoniae clinical isolates, the quercetin/colistin combination also showed a great synergy effect in eradicating the infected bacteria. Taken together, our work is of great significance for the clinical treatment of refractory colistin-resistant bacteria infections via teaching an old dog a new trick.

\section{DATA AVAILABILITY STATEMENT}

The original contributions presented in the study are included in the article/Supplementary Material, further inquiries can be directed to the corresponding authors.

\section{REFERENCES}

Arias, C. A., and Murray, B. E. (2012). The Rise of the Enterococcus: beyond Vancomycin Resistance. Nat. Rev. Microbiol. 10 (4), 266-278. doi:10.1038/ nrmicro2761

Berard, X., Stecken, L., Pinaquy, J.-B., Cazanave, C., Puges, M., Pereyre, S., et al. (2016). Comparison of the Antimicrobial Properties of Silver Impregnated Vascular Grafts with and without Triclosan. Eur. J. Vasc. Endovascular Surg. 51 (2), 285-292. doi:10.1016/j.ejvs.2015.10.016

Biswas, S., Brunel, J.-M., Dubus, J.-C., Reynaud-Gaubert, M., and Rolain, J.-M. (2014). Colistin: an Update on the Antibiotic of the 21st century. Expert Rev. Anti-infective Ther. 10 (8), 917-934. doi:10.1586/eri.12.78

Bulman, Z. P., Chen, L., Walsh, T. J., Satlin, M. J., Qian, Y., Bulitta, J. B., et al. (2017). Polymyxin Combinations Combat Escherichia coli Harboring Mcr-1 and Bla NDM-5 : Preparation for a Postantibiotic Era. mBio 8 (4), e00540-17. doi:10.1128/mBio.00540-17

Córdoba, J., Coronado-Álvarez, N. M., Parra, D., and Parra-Ruiz, J. (2015). In VitroActivities of Novel Antimicrobial Combinations against Extensively Drug-Resistant Acinetobacter Baumannii. Antimicrob. Agents Chemother. 59 (12), 7316-7319. doi:10.1128/AAC.00493-15

Gaudereto, J. J., Perdigão Neto, L. V., Leite, G. C., Ruedas Martins, R., Boas do Prado, G. V., Rossi, F., et al. (2019). Synergistic Effect of Ceftazidime-Avibactam with Meropenem against Panresistant, Carbapenemase-Harboring Acinetobacter Baumannii and Serratia marcescens Investigated Using TimeKill and Disk Approximation Assays. Antimicrob. Agents Chemother. 63 (5), e02367. doi:10.1128/AAC.02367-18

Grégoire, N., Aranzana-Climent, V., Magréault, S., Marchand, S., and Couet, W. (2017). Clinical Pharmacokinetics and Pharmacodynamics of Colistin. Clin. Pharmacokinet. 56 (12), 1441-1460. doi:10.1007/s40262-017-0561-1

Hao, Y., Zheng, W., Sun, Z., Zhang, D., Sui, K., Shen, P., et al. (2021). Marine Polysaccharide-Based Composite Hydrogels Containing Fucoidan: Preparation, Physicochemical Characterization, and Biocompatible Evaluation. Int. J. Biol. Macromolecules 183, 1978-1986. doi:10.1016/ j.ijbiomac.2021.05.190

Jaisinghani, R. N. (2017). Antibacterial Properties of Quercetin. Microbiol. Res. (Pavia) 8 (1), 13-14. doi:10.4081/mr.2017.6877

Jayol, A., Poirel, L., Brink, A., Villegas, M.-V., Yilmaz, M., and Nordmann, P. (2014). Resistance to Colistin Associated with a Single Amino Acid Change in Protein PmrB Among Klebsiella pneumoniae Isolates of Worldwide Origin. Antimicrob. Agents Chemother. 58 (8), 4762-4766. doi:10.1128/AAC.00084-14

\section{ETHICS STATEMENT}

The animal study was reviewed and approved by the ethics committee of the Wenzhou Medical University (SYXK 20200014).

\section{AUTHOR CONTRIBUTIONS}

All authors contributed to the study's conception and design. Material preparation and data collection were performed by YZ, SL, and NH. Data analysis was performed by LC, WZ, and WL. YZ, TZ, and JC supervised the research. The first draft of the manuscript was written by YL. All authors contributed to the revisions of the manuscript and approved the final manuscript.

\section{FUNDING}

The authors acknowledge the financial support of the Planned Science and Technology Project of Wenzhou (no. Y20170204).

Jiang, Y., Zhang, Y., Lu, J., Wang, Q., Cui, Y., Wang, Y., et al. (2020). Clinical Relevance and Plasmid Dynamics of Mcr-1-Positive Escherichia coli in China: a Multicentre Case-Control and Molecular Epidemiological Study. The Lancet Microbe 1 (1), e24-e33. doi:10.1016/s2666-5247(20)30001-x

Li, Y. F., Liu, Y., Ren, Y. J., Su, L. Z., Li, A., An, Y. L., et al. (2020). Coating of a Novel Antimicrobial Nanoparticle with a Macrophage Membrane for the Selective Entry into Infected Macrophages and Killing of Intracellular Staphylococci. Adv. Funct. Mater. 30 (48), 1-16. doi:10.1002/adfm.202004942

Liao, W., Lin, J., Jia, H., Zhou, C., Zhang, Y., Lin, Y., et al. (2020). Resistance and Heteroresistance to Colistin in Escherichia coli Isolates from Wenzhou, China. Infect. Drug Resist. 13, 3551-3561. doi:10.2147/IDR.S273784

Liu, Y.-Y., Wang, Y., Walsh, T. R., Yi, L.-X., Zhang, R., Spencer, J., et al. (2016). Emergence of Plasmid-Mediated Colistin Resistance Mechanism MCR-1 in Animals and Human Beings in China: a Microbiological and Molecular Biological Study. Lancet Infect. Dis. 16 (2), 161-168. doi:10.1016/s14733099(15)00424-7

Liu, Y., Ding, S., Dietrich, R., Märtlbauer, E., and Zhu, K. (2017). A BiosurfactantInspired Heptapeptide with Improved Specificity to Kill MRSA. Angew. Chem. Int. Ed. 56 (6), 1486-1490. doi:10.1002/anie.201609277

Liu, Y., Li, Y., and Shi, L. (2021). Controlled Drug Delivery Systems in Eradicating Bacterial Biofilm-Associated Infections. J. Controlled Release 329, 1102-1116. doi:10.1016/j.jconrel.2020.10.038

Pal, A., and Tripathi, A. (2020). Quercetin Inhibits Carbapenemase and Efflux Pump Activities Among Carbapenem-resistant Gram-negative Bacteria. APMIS 128 (3), 251-259. doi:10.1111/apm.13015

Pal, A., and Tripathi, A. (2019). Quercetin Potentiates Meropenem Activity Among Pathogenic carbapenem-resistantPseudomonas aeruginosaandAcinetobacter Baumannii. J. Appl. Microbiol. 127 (4), 1038-1047. doi:10.1111/jam.14388

Phan, M.-D., Nhu, N. T. K., Achard, M. E. S., Forde, B. M., Hong, K. W., Chong, T. M., et al. (2017). Modifications in the pmrB Gene Are the Primary Mechanism for the Development of Chromosomally Encoded Resistance to Polymyxins in Uropathogenic Escherichia coli. J. Antimicrob. Chemother. 72 (10), 2729-2736. doi:10.1093/jac/dkx204

Qu, S., Dai, C., Shen, Z., Tang, Q., Wang, H., Zhai, B., et al. (2019). Mechanism of Synergy between Tetracycline and Quercetin against Antibiotic Resistant Escherichia coli. Front. Microbiol. 10, 2536. doi:10.3389/fmicb.2019.02536

Reyes-Farias, M., and Carrasco-Pozo, C. (2019). The Anti-cancer Effect of Quercetin: Molecular Implications in Cancer Metabolism. Int. J. Mol Sci. 20 (13), 3177. doi:10.3390/ijms20133177

Shen, C., Feng, S., Chen, H., Dai, M., Paterson, D. L., Zheng, X., et al. (2018). Transmission Ofmcr-1-Producing Multidrug-Resistant Enterobacteriaceae in 
Public Transportation in Guangzhou, China. Clin. Infect. Dis. 67 (Suppl. 1_2), S217-S224. doi:10.1093/cid/ciy661

Shen, Y., Zhang, R., Schwarz, S., Wu, C., Shen, J., Walsh, T. R., et al. (2020). Farm Animals and Aquaculture: Significant Reservoirs of mobile Colistin Resistance Genes. Environ. Microbiol. 22 (7), 2469-2484. doi:10.1111/1462-2920.14961

Su, L.-Z., Liu, Y., Li, Y.-F., An, Y.-L., and Shi, L.-Q. (2021). Responsive Polymeric Nanoparticles for Biofilm-Infection Control. Chin. J. Polym. Sci. 39, 1376-1391. doi:10.1007/s10118-021-2610-3

Su, L., Li, Y., Liu, Y., Ma, R., Liu, Y., Huang, F., et al. (2020). Antifungal-Inbuilt Metal-Organic-Frameworks Eradicate Candida Albicans Biofilms. Adv. Funct. Mater. 30 (28), 1-11. doi:10.1002/adfm.202000537

Tadesse, D. A., Zhao, S., Tong, E., Ayers, S., Singh, A., Bartholomew, M. J., et al. (2012). Antimicrobial Drug Resistance inEscherichia Colifrom Humans and Food Animals, United States, 1950-2002. Emerg. Infect. Dis. 18 (5), 741-749. doi:10.3201/eid1805.111153

Tran, T. B., Wang, J., Doi, Y., Velkov, T., Bergen, P. J., and Li, J. (2018). Novel Polymyxin Combination with Antineoplastic Mitotane Improved the Bacterial Killing against Polymyxin-Resistant Multidrug-Resistant Gram-Negative Pathogens. Front. Microbiol. 9, 721. doi:10.3389/fmicb.2018.00721

Vargas, A. J., and Burd, R. (2010). Hormesis and Synergy: Pathways and Mechanisms of Quercetin in Cancer Prevention and Management. Nutr. Rev. 68 (7), 418-428. doi:10.1111/j.1753-4887.2010.00301.x

Vilaça, R., Mendes, V., Mendes, M. V., Carreto, L., Amorim, M. A., de Freitas, V., et al. (2012). Quercetin Protects Saccharomyces cerevisiae against Oxidative Stress by Inducing Trehalose Biosynthesis and the Cell wall Integrity Pathway. PLoS One 7 (9), e45494. doi:10.1371/journal.pone.0045494

Vipin, C., Saptami, K., Fida, F., Mujeeburahiman, M., Rao, S. S., Athmika, et al. (2020). Potential Synergistic Activity of Quercetin with Antibiotics against Multidrug-Resistant Clinical Strains of Pseudomonas aeruginosa. PLoS One 15 (11), e0241304. doi:10.1371/journal.pone.0241304

Watanabe, G., Sekiya, H., Tamai, E., Saijo, R., Uno, H., Mori, S., et al. (2018). Synthesis and Antimicrobial Activity of 2-Trifluoroacetonylbenzoxazole Ligands and Their Metal Complexes. Chem. Pharm. Bull. 66 (7), 732-740. doi:10.1248/cpb.c18-00158

Xavier, B. B., Lammens, C., Ruhal, R., Kumar-Singh, S., Butaye, P., Goossens, H., et al. (2016). Identification of a Novel Plasmid-Mediated Colistin-Resistance Gene, Mcr-2, in Escherichia coli, Belgium, June 2016. Euro Surveill. 21 (27), 1-6. doi:10.2807/1560-7917.ES.2016.21.27.30280
Yu, Y., Huang, H.-L., Ye, X.-Q., Cai, D.-T., Fang, J.-T., Sun, J., et al. (2020) Synergistic Potential of Antimicrobial Combinations against MethicillinResistant Staphylococcus aureus. Front. Microbiol. 11, 1919. doi:10.3389/ fmicb.2020.01919

Zhang, Y., Wang, X., Li, X., Dong, L., Hu, X., Nie, T., et al. (2019). Synergistic Effect of Colistin Combined with PFK-158 against Colistin-Resistant Enterobacteriaceae. Antimicrob. Agents Chemother. 63 (7), e00271-19. doi:10.1128/AAC.00271-19

Zhao, W.-H., Hu, Z.-Q., Okubo, S., Hara, Y., and Shimamura, T. (2001). Mechanism of Synergy between Epigallocatechin Gallate and $\beta$-Lactams against Methicillin-Resistant Staphylococcus aureus. Antimicrob. Agents Chemother. 45 (6), 1737-1742. doi:10.1128/AAC.45.6.1737-1742.2001

Zhou, Y.-F., Tao, M.-T., Feng, Y., Yang, R.-S., Liao, X.-P., Liu, Y.-H., et al. (2017). Increased Activity of Colistin in Combination with Amikacin against Escherichia coli Co-producing NDM-5 and MCR-1. J. Antimicrob. Chemother. 72 (6), 1723-1730. doi:10.1093/jac/dkx038

Zhu, Y., Liu, L., Sun, Z., Ji, Y., Wang, D., Mei, L., et al. (2021). Fucoidan as a marineorigin Prebiotic Modulates the Growth and Antibacterial Ability of Lactobacillus Rhamnosus. Int. J. Biol. Macromolecules 180, 599-607. doi:10.1016/j.ijbiomac.2021.03.065

Conflict of Interest: The authors declare that the research was conducted in the absence of any commercial or financial relationships that could be construed as a potential conflict of interest.

Publisher's Note: All claims expressed in this article are solely those of the authors and do not necessarily represent those of their affiliated organizations, or those of the publisher, the editors and the reviewers. Any product that may be evaluated in this article, or claim that may be made by its manufacturer, is not guaranteed or endorsed by the publisher.

Copyright (® 2021 Lin, Zhang, Liu, Ye, Chen, Huang, Zeng, Liao, Zhan, Zhou and Cao. This is an open-access article distributed under the terms of the Creative Commons Attribution License (CC BY). The use, distribution or reproduction in other forums is permitted, provided the original author(s) and the copyright owner(s) are credited and that the original publication in this journal is cited, in accordance with accepted academic practice. No use, distribution or reproduction is permitted which does not comply with these terms. 